\title{
Folistatina, resistencia a la insulina y composición corporal en adultos colombianos
}

\author{
Toloza FJK, $\mathrm{MD}^{1}$; Ricardo-Silgado $\mathrm{ML}, \mathrm{MD}^{1}$; Mantilla-Rivas JO, $\mathrm{MD}^{1}$; Morales-Álvarez \\ $M C, M D^{1}$; Pérez-Matos $M C, M D^{1}$; Pinzón-Cortés JA, $M D, M S c^{1}$; Pérez-Mayorga $M, M^{2}$, \\ Mendivil $C O, M D, P h D^{1,3}$.
}

\footnotetext{
${ }^{1}$ Universidad de los Andes, Facultad de Medicina, Bogotá, Colombia.

${ }^{2}$ Universidad Militar Nueva Granada, Facultad de Medicina, Bogotá, Colombia.

${ }^{3}$ Fundación Santa Fe de Bogotá, Sección de Endocrinología, Bogotá, Colombia.
}

Autor de correspondencia:

Carlos O. Mendivil

Carrera 7 No 116-05, of 413

Facultad de Medicina, Universidad de los Andes, Bogotá, Colombia. Email: carlosolimpo@gmail.com o cmendivi@uniandes.edu.co Declaración de fuentes de financiación:

Este estudio fue patrocinado por el Departamento Administrativo de Ciencia, Tecnología e Innovación de Colombia, COLCIENCIAS y la Vicerrectoría de Investigaciones de la Universidad de los Andes.

Conflictos de interés reportados: Ninguno.

Fecha de recepción: 24/10/2017

Fecha de aceptación: 6/12/2017

\section{Resumen}

Introducción: La folistatina es una proteína capaz de neutralizar varias hormonas de la familia del TGF- $\beta$, tales como la activina, las proteínas morfogénicas del hueso y la miostatina. $\mathrm{Al}$ inactivar la activina y la folistatina reduce la secreción de FSH. La folistatina se produce además de en el ovario en muchos otros tejidos, por ello se sospecha que tiene otros efectos. En ratones, la deleción genética de la folistatina se acompaña de resistencia a la insulina (RI). Sin embargo, la asociación entre la folistatina plasmática y RI medida directamente no ha sido evaluada en humanos.

Métodos: En 81 participantes entre 30 y 69 años (56\% mujeres, $54 \%$ con sobrepeso, $13 \%$ con obesidad), determinamos antropometría, composición corporal, factores de riesgo cardiovascular y múltiples índices de RI: Área incremental bajo la curva de insulina, índice de sensibilidad a la insulina según Gutt, Homeostatic Model Assessment - Insulin Resistance (HOMA-IR) e insulinemia en ayuno. Un subgrupo de 21 participantes se sometió además a un clamp hiperinsulinémico-eu- glucémico. La folistatina y la miostatina se midieron en plasma de ayuno, empleando técnicas inmunométricas.

Resultados: La concentración promedio de folistatina fue $2.517 \pm 830 \mathrm{pg} / \mathrm{mL}$, sin diferencia entre sexos $(\mathrm{p}=0,55)$. La folistatina tuvo una tendencia a correlación positiva con el porcentaje de masa magra $(r=0,19, p=0,088)$ y negativa con el porcentaje de grasa corporal $(r=-0,19, p=0,097)$. La folistatina no se correlacionó con índices de RI derivados de la PTOG pero sí con la captación corporal de glucosa en el clamp $(\mathrm{r}=0,42$, $\mathrm{p}=0,031)$. No se halló asociación entre las concentraciones de folistatina y miostatina plasmáticas.

Conclusión: Los niveles de folistatina mostraron una tendencia hacia una correlación positiva con la masa muscular y negativa con adiposidad corporal. Esto concuerda con el efecto inhibitorio de la folistatina sobre la miostatina. Aunque la folistatina no correlacionó con índices indirectos de RI, sí lo hizo con la determinación directa de sensibilidad a la insulina en el clamp hiperinsulinémico-euglucémico.

Palabras clave: folistatina, resistencia a la insulina, obesidad, adiposidad, miostatina, miocinas, TGF

\section{Abstract}

Introduction: Follistatin is a protein with the ability to neutralize several hormones of the TGF-beta family including activin, bone morphogenetic proteins and myostatin. By inactivating activin, follistatin reduces FSH secretion. Besides ovary, follistatin is produced in several other tissues, hence it may have many other effects. In mice, genetic follistatin defficiency leads to insulin resistance (IR). However, the association between plasma follistatin and directly measured IR has not been evaluated in humans.

Methods: We determined in 81 participants aged 30 to 69 (56\% women, 54\% overweight, 13\% obese) anthropometry, body composition, cardiovascular risk factors and multiple IR indices: Incremental area under the insulin curve, Gutt's Insulin sensitivity index, homeostatic model assessment - insulin resistance (HOMA-IR) and fasting insulin. A subgroup of 21 participants additionally underwent a hyperinsulinemic/euglycemic 
clamp. Follistatin and myostatin were measured in fasting plasma using immunometric techniques.

Results: Mean plasma follistatin was $2.517 \pm 830 \mathrm{pg} / \mathrm{mL}$, without differences by sex $(p=0,55)$. Follistatin showed a trend towards a positive correlation with the percent lean mass $(r=0,19$, $p=0,088$ ) and towards a negative correlation with percent body fat $(r=-0,19, p=0,097)$. Follistatin was not correlated with OGTTderived IR indices but it did correlate with whole body insulinstimulated glucose uptake in the clamp $(r=0,42, p=0,031)$. No correlation existed between plasma follistatin and myostatin.

Conclusion: Plasma follistatin showed a trend to a positive correlation with muscle mass, and negative with fat mass. This fits the known role of follistatin as an antagonist to myostatin. Even though follistatin was not associated with indirect IR indices, it was positively associated with directly measured insulin sensitivity in the clamp.

Keywords: follistatin, insulin resistance, obesity, adiposity, myostatin, myokines, TGF

\section{Introducción}

La resistencia a la insulina (RI) es el mecanismo fisiopatológico central de la diabetes mellitus tipo 2 (DM2). Sin embargo, para el diagnóstico preciso de la RI se requiere de métodos sofisticados y costosos, que dan como resultado la detección tardía de este fenómeno. El patrón de oro para la detección de RI es el clamp euglucémico hiperinsulinémico, un procedimiento invasivo y poco práctico, siendo así inadecuado para uso rutinario. Los avances recientes en proteómica han identificado posibles biomarcadores tempranos de RI - uno de ellos es la folistatina ${ }^{(1,2)}$ - pero no se conoce completamente su asociación con RI en diferentes poblaciones.

La folistatina fue identificada en 1987 como una proteína soluble presente en el fluido ovárico porcino ${ }^{(3)}$. Inicialmente su función fue relacionada con la supresión de la hormona estimulante del folículo (FSH), y debido a eso recibe su nombre ${ }^{(4)}$.
No obstante, en estudios posteriores se encontró que la mayor parte de la folistatina en la circulación proviene del hígado, de forma que su función no está completamente entendida ${ }^{(5)}$.

En humanos, la folistatina se encuentra codificada en el cromosoma $5 q 11.2^{(6)}$ y dentro de su estructura contiene un sitio de unión a TGF- $\beta$, lo que le confiere la propiedad de unirse y neutralizar a varias hormonas (todas pertenecientes a la familia del TGF- $\beta$ ) como la activina ${ }^{(7)}$, la miostatina ${ }^{(8)}$ y las proteínas morfogénicas del hueso ${ }^{(9)}$. La folistatina existe en dos isoformas: folistatina 288 y folistatina 315 , nombradas de acuerdo al número de aminoácidos que contienen ${ }^{(10)}$. La diferencia entre las dos radica en un segmento C-terminal adicional de 27 aminoácidos que contiene la isoforma 315 , que le impide interactuar con los proteoglicanos extracelulares ${ }^{(11,12)}$. Así, la folistatina 315 es la forma más abundante en la circulación, mientras que la folistatina 288 es la forma predominantemente tisular ${ }^{(12)}$.

Estudios en modelos animales han mostrado que los niveles plasmáticos de folistatina se encuentran elevados en diferentes situaciones fisiológicas como el ayuno prolongado ${ }^{(13)} \mathrm{y}$ el ejercicio ${ }^{(14,15)}$. Estas dos situaciones tienen en común niveles aumentados de glucagón y disminución de la insulina, como una medida para la movilización de la energía almacenada. Así, se ha propuesto que la secreción hepática de la folistatina es regulada por la relación glucagón/insulina ${ }^{(16,17)}$. De esta forma, la concentración sérica de la folistatina podría funcionar como una señal de deprivación energética ${ }^{(1)}$. Por otra parte, y debido a su efecto en la inhibición de la miostatina, el incremento de folistatina puede constituir mecanismo de protección muscular durante el ayuno ${ }^{(8)}$ (figura 1).

En humanos, existen pocos estudios que han evaluado la correlación de la folistatina con las alteraciones del metabolismo. En pacientes con DM2, los niveles plasmáticos de folistatina son mayores comparados con controles sanos, y se asocian con la glucemia basal y la hemoglobina glucosilada ${ }^{(2)}$. En sujetos sanos, por el contrario, la relación entre hiperglucemia y

Figura 1. Mecanismo de regulación y fisiología en la secreción de folistatina

\section{Ayuno prolongado Ejercicio}

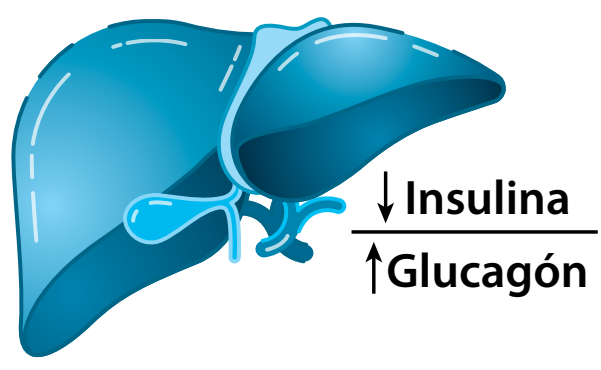

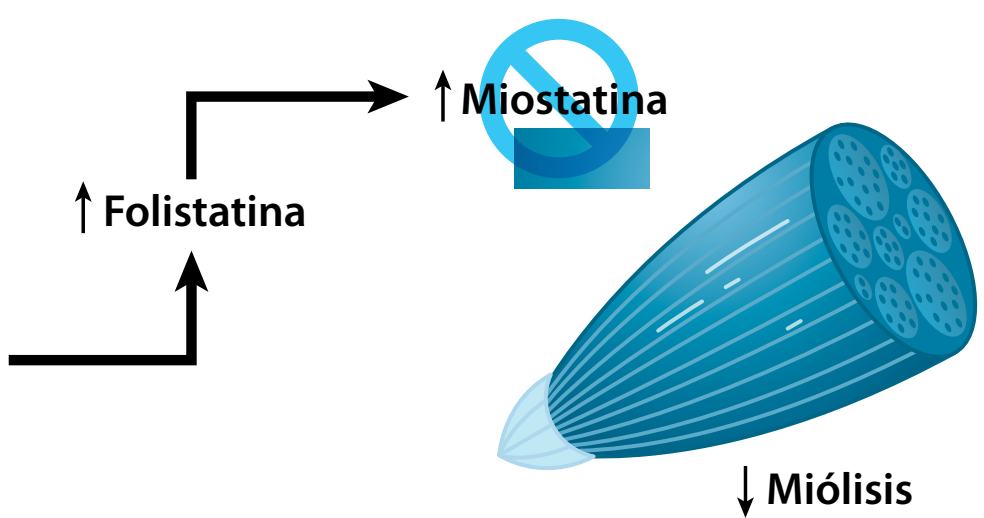


folistatina ha resultado significativa ${ }^{(16,17)}$. Durante el ejercicio agudo, los individuos con DM2 presentan una respuesta anormalmente baja en la secreción de folistatina ${ }^{(17)}$.

La evidencia respecto a la asociación de la folistatina y los trastornos del metabolismo en humanos es escasa y contradictoria y no hay estudios que hayan explorado el papel de la folistatina en la RI medida directamente. El objetivo de este estudio fue evaluar la asociación entre los niveles plasmáticos de folistatina con la RI medida de diferentes maneras en una muestra de participantes sin diagnóstico previo de DM2.

\section{Métodos}

\section{Muestra}

Se incluyeron 81 adultos entre 30 y 69 años, sin diagnóstico previo de DM2, residentes de Bogotá, reclutados entre abril de 2015 y octubre de 2016. Con el fin de incluir participantes con grados de sensibilidad a la insulina presumiblemente diferentes, seleccionamos intencionalmente individuos de ambos sexos con diferentes índices de masa corporal (IMC).

Todos los sujetos manifestaron su deseo de participar y proporcionaron su consentimiento informado por escrito. Se excluyeron personas con diagnóstico previo de diabetes mellitus o que estuvieran en manejo con medicamentos antidiabéticos, con enfermedades del páncreas exocrino, mujeres embarazadas o pacientes que recibieron anticoagulantes orales. También se excluyeron los pacientes que presentaran síntomas de una infección viral o bacteriana aguda o que tuviesen niveles de proteína $\mathrm{C}$ reactiva de alta sensibilidad (hsPCR) superiores a $10 \mathrm{mg} / \mathrm{L}$.

\section{Mediciones clínicas}

Realizamos un examen físico completo con medición de la presión arterial, antropometría y composición corporal (porcentaje de grasa corporal, porcentaje de grasa abdominal, agua corporal total, grasa corporal segmentaria) evaluada por impedanciometría tetrapolar (monitor de composición corporal IRONMAN BC545, TANITA). Tomamos muestras de sangre en ayunas en tubos con fluoruro de sodio/oxalato de potasio para la determinación de la glucemia basal y en EDTA para todas las demás mediciones de laboratorio (insulina, creatinina, perfil de lípidos, hsPCR, folistatina y miostatina). El plasma se separó rápidamente, se dividió en alícuotas en tubos separados para cada medición y se congeló a $-80^{\circ} \mathrm{C}$ hasta su determinación.

Después de un ayuno de 8 a 12 h, los pacientes realizaron una prueba de tolerancia oral a la glucosa (PTOG) con una carga de $75 \mathrm{~g}$ de glucosa diluida en $300 \mathrm{~mL}$ de agua, que tuvieron que consumir en menos de 5 minutos. Se obtuvieron muestras de plasma para medir la glucosa y la insulina justo antes de la ingestión de la carga de glucosa (tiempo 0), y a los 30, 60, 90 y 120 minutos después de la carga de glucosa. Los pacientes permanecieron sentados y no pudieron fumar, ingerir alimentos o bebidas durante el procedimiento.
Un subgrupo seleccionado al azar de 21 participantes se sometió además a un clamp euglucémico hiperinsulinémico, de acuerdo con lo descrito por deFronzo ${ }^{(18)}$. Después de 8 horas de ayuno, los sujetos fueron ingresados en un centro clínico ambulatorio, donde se colocaron dos catéteres IV en el área antecubital de ambos brazos para la infusión de insulina y de dextrosa. Se colocó otro catéter en la zona dorsal de la mano ipsilateral a la infusión de dextrosa para la toma de muestras de sangre. La mano de la cual se tomaron las muestras de sangre capilar para la determinación de la glucemia se colocó dentro de una caja de calentamiento (heated-hand box) (University of Vermont, EE.UU.). Este dispositivo permite el mantenimiento de una temperatura controlada constante $\left(56-58^{\circ} \mathrm{C}\right)$ para arterializar la sangre venosa.

Se infundió un análogo de insulina humano de acción corta (Humalog, Lilly) a una velocidad inicial de $100 \mathrm{mUI} / \mathrm{m}^{2} / \mathrm{min}$ que se redujo a $90 \mathrm{mUI} / \mathrm{m}^{2} / \mathrm{min}$ después de 2 minutos y luego fue reducida en $20 \mathrm{mUI} / \mathrm{m}^{2} / \mathrm{min}$ cada 2 minutos hasta que se alcanzó una dosis de $40 \mathrm{mUI} / \mathrm{m}^{2} / \mathrm{min}$. Esta tasa se mantuvo constante durante los siguientes 110 minutos, para completar 2 horas de procedimiento. Adicionalmente, se inició una infusión variable de $20 \%$ de glucosa a los 4 minutos del inicio del clamp y se ajustó cada 5 minutos para mantener la concentración de glucosa venosa arterializada en 100 mg/dL (95-105 $\mathrm{mg} / \mathrm{dL}$ ). La glucosa plasmática se midió mediante el método de la glucosa oxidasa, usando el glucómetro Accu-Chek Performa (Roche, Alemania). El resultado principal del clamp fue la captación corporal de glucosa en estado de equilibrio (M) (mg de glucosa/ kg de peso corporal/min).

\section{Mediciones bioquímicas}

Medimos la glucemia basal, los lípidos plasmáticos y la creatinina utilizando ensayos colorimétricos convencionales (Biosystems, España). La hemoglobina glucosilada A1c (HbA1c) se determinó usando una técnica de afinidad de boronato certificada por el Programa Nacional de Normalización de Hemoglobina glucosilada (NycoCard ${ }^{\circledR}$ Reader II, Alere Technologies, Noruega). Se midió la folistatina plasmática usando el kit R\&D Systems Human Follistatin Quantikine ELISA (CAT\#DFN00, rango dinámico $250-16000$ pg/mL), la miostatina usando el kit R\&D Systems GDF-8/Myostatin Quantikine ELISA (CAT\#DGDF80, rango dinámico 31,3 - 2000 pg/mL) y la hsCRP con el kit R\&D Systems Human C-Reactive Protein/CRP Immunoassay (CAT\#DCRP00, rango dinámico 0.010-50 ng/ $\mathrm{mL}$ ). Medimos la insulinemia basal y todas las demás mediciones de insulina derivadas de la PTOG con el kit de ELISA Abcam Insulin Human (CAT\#ab100578, rango dinámico 4.7-300 microUI/mL). Todos los inmunoensayos se basaron en metodologías de sándwich simple o doble. Las absorbancias se determinaron con el BioTek Synergy HT Reader ${ }^{\circledR}$ a la longitud de onda óptima del cromógeno empleado en cada prueba. Realizamos todas las mediciones por duplicado, y ejecutamos con- 
troles positivos y negativos dentro de cada lote. Ejecutamos todos los procedimientos en el Laboratorio de Diabetes, Lípidos y Metabolismo de la Universidad de los Andes, siguiendo los protocolos institucionales actuales de bioseguridad.

\section{Análisis estadísticos}

Los índices de RI derivados de los valores plasmáticos de glucosa e insulina en la PTOG fueron:

- Homeostasis Model Assessment of Insulin Resistance (HOMA-IR) ${ }^{(19)}$ :

HOMA-IR: [Insulinemia basal (microU/mL) * Glucemia basal (mmol/L)] /22,5.

- Índice de sensibilidad a la insulina por Gutt (ISI-Gutt) ${ }^{(20)}$ : ISI: $(\{75000+[$ Glucemia basal (mg/L) - Glucemia a los 120 $\min (\mathrm{mg} / \mathrm{L})]\} * 0,19 *$ peso corporal $(\mathrm{Kg}) / 120 \mathrm{~min} /$ [Promedio de glucemia durante la PTOG (mg/L)] / [log promedio de la insulinemia durante la PTOG (microU $/ \mathrm{mL})]$.

- Respuesta corregida a la insulina a los 30 minutos (CIR$30)^{(19):}$

CIR-30: (100* Insulina a los $30 \mathrm{~min}$ ) / \{Glucemia a los $30 \mathrm{~min}$ (mg/dL) * [Glucemia a los $30 \mathrm{~min}(\mathrm{mg} / \mathrm{dL})-70(\mathrm{mg} / \mathrm{dL})]\}$

- Quantitative insulin sensitivity check index (QUICKI) ${ }^{(19)}$ :

QUICKI: 1 / (Glucemia basal [mmol/L] + Insulinemia basal $[\mathrm{microU} / \mathrm{mL}])$.

- Área incremental bajo la curva de insulina (iAUCins), calculada por el método del trapezoide ${ }^{(21)}$.

Todos los análisis se realizaron con Statistical Package for Social Sciences versión 19,0 (SPSS, Inc, Chicago, IL, USA). Las correlaciones entre los índices RI y la folistatina plasmática, y entre las variables clínicas y los niveles de folistatina se examinaron utilizando los coeficientes de correlación de Spearman. Las asociaciones que implican el valor M también se exploraron con los coeficientes de correlación de Spearman. Los participantes se distribuyeron en cuartiles de acuerdo con los valores de los índices RI (HOMA-IR, ISI-Gutt, iAUCins, CIR-30 y QUICKI). Las concentraciones medias de cada biomarcador se calcularon para cada cuartil de cada predictor. Los valores de $p$ de tendencia reportados ( $p$-trend) corresponden a los asociados con el coeficiente de la pendiente en una regresión lineal en la que los valores medios en cada uno de los cuartiles de IR fueron las variables independientes y las concentraciones medias de folistatina en cada cuartil fueron la variable dependiente. Todas las pruebas estadísticas fueron de dos colas a un nivel de significancia de 0,05.

Se utilizó la relación de glucemia basal/insulinemia basal (G/I) como un subrogado de la relación glucagón/insulina.

\section{Resultados}

La muestra estuvo compuesta en un $43 \%$ por hombres y $57 \%$ mujeres. La media de edad fue $51,4 \pm 10,1$ años, IMC $26,7 \mathrm{~kg} / \mathrm{m}^{2}$ (rango 16,0-39,9) y circunferencia abdominal
95,1 \11,6 cm (rango 64-130). La presión arterial media, el colesterol total, el LDLc y la creatinina estuvieron en promedio dentro del rango normal. Las características demográficas, clínicas y bioquímicas de los participantes se encuentran resumidas en la tabla 1.

\section{Folistatina y variables clínicas}

La concentración promedio de folistatina fue $2.517 \pm 830$ $\mathrm{pg} / \mathrm{mL}$, sin diferencia entre sexos $(\mathrm{p}=0,55)$. La folistatina tuvo una tendencia a presentar una correlación positiva con el porcentaje de masa magra $(r=0,19, p=0,088)$ y negativa con el porcentaje de grasa corporal $(r=-0,19, p=0,097)$ (figura 2).

Tabla 1. Características demográficas, clínicas y bioquímicas de los participantes

\begin{tabular}{|c|c|c|c|}
\hline & $\begin{array}{l}\text { Mujeres } \\
(n=46)\end{array}$ & $\begin{array}{c}\text { Hombres } \\
(n=35)\end{array}$ & $\begin{array}{c}\text { Total } \\
(n=81)\end{array}$ \\
\hline Edad (años) & $52,6 \pm 8,5$ & $50,9 \pm 11,8$ & $51,4 \pm 10,1$ \\
\hline Peso (Kg) & $63,5 \pm 11,0$ & $78,7 \pm 13,2$ & $70,4 \pm 13,8$ \\
\hline Estatura $(\mathrm{cm})$ & $156,9 \pm 5,9$ & $168,6 \pm 7,8$ & $161,9 \pm 8,9$ \\
\hline $\begin{array}{l}\text { Índice de masa } \\
\text { corporal }\left(\mathrm{Kg} / \mathrm{m}^{2}\right)\end{array}$ & $25,8 \pm 4,3$ & $27,6 \pm 4$ & $26,7 \pm 4,1$ \\
\hline Grasa corporal (\%) & $34,7 \pm 5,8$ & $26,4 \pm 6,2$ & $31,1 \pm 7,3$ \\
\hline $\begin{array}{l}\text { Circunferencia } \\
\text { abdominal }(\mathrm{cm})\end{array}$ & $82,5 \pm 10,5$ & $95,1 \pm 11,6$ & $88,2 \pm 12,7$ \\
\hline $\begin{array}{l}\text { Tensión arterial } \\
\text { sistólica }(\mathbf{m m H g})\end{array}$ & $114,9 \pm 14,1$ & $121,7 \pm 16,6$ & $118 \pm 15,4$ \\
\hline $\begin{array}{l}\text { Tensión arterial } \\
\text { diastólica }(\mathbf{m m H g})\end{array}$ & $73,2 \pm 10,4$ & $75,3 \pm 10,2$ & $71,4 \pm 17,3$ \\
\hline $\begin{array}{l}\text { Glucemia de ayuno } \\
\text { (mg/dL) }\end{array}$ & $94,3 \pm 11,5$ & $98,1 \pm 6,6$ & $95,9 \pm 9,8$ \\
\hline $\begin{array}{l}\text { Hemoglobina } \\
\text { glucosilada } \\
\text { (HbA1c) (\%) }\end{array}$ & $5,7 \pm 1,6$ & $5,6 \pm 1,0$ & $5,5 \pm 1,4$ \\
\hline Creatinina $(\mathrm{mg} / \mathrm{dL})$ & $0,73 \pm 0,12$ & $0,92 \pm 0,17$ & $0,81 \pm 0,17$ \\
\hline $\begin{array}{l}\text { Tasa de filtración } \\
\text { glomerular (mL/ } \\
\text { min/1,73m²) }\end{array}$ & $92,3 \pm 16,6$ & $96,2 \pm 22,2$ & $94,1 \pm 19,3$ \\
\hline $\begin{array}{l}\text { Triglicéridos } \\
\text { (mg/dL) }\end{array}$ & $153,9 \pm 84$ & $156,4 \pm 68,2$ & $154,6 \pm 77,1$ \\
\hline $\begin{array}{l}\text { Colesterol HDL } \\
\text { (mg/dL) }\end{array}$ & $47,8 \pm 11,3$ & $39,0 \pm 11,9$ & $43,9 \pm 12,3$ \\
\hline $\begin{array}{l}\text { Colesterol LDL } \\
\text { (mg/dL) }\end{array}$ & $134,4 \pm 46,8$ & $112,5 \pm 41$ & $\begin{array}{c}125,2 \pm \\
45,2\end{array}$ \\
\hline $\begin{array}{l}\text { Proteína C reactiva } \\
(\mathrm{PCR})(\mathrm{mg} / \mathrm{L})\end{array}$ & $2,08 \pm 1,85$ & $3,35 \pm 6,8$ & $2,54 \pm 4,27$ \\
\hline Folistatina (ng/mL) & $\begin{array}{c}2492,0 \pm \\
777,3\end{array}$ & $\begin{array}{c}2603,6 \pm \\
861,1 \\
\end{array}$ & $\begin{array}{c}2517,0 \pm \\
830,0\end{array}$ \\
\hline Miostatina (ng/mL) & $\begin{array}{c}2494,3 \pm \\
1670,4\end{array}$ & $\begin{array}{c}3552,8 \pm \\
2034,6\end{array}$ & $\begin{array}{c}2957,8 \pm \\
1901,2\end{array}$ \\
\hline
\end{tabular}

Los datos son expresados como media $\pm \mathrm{DE}$ 
Figura 2. Correlación entre porcentaje de grasa corporal (izquierda) o porcentaje de masa magra corporal (derecha) y niveles séricos de folistatina

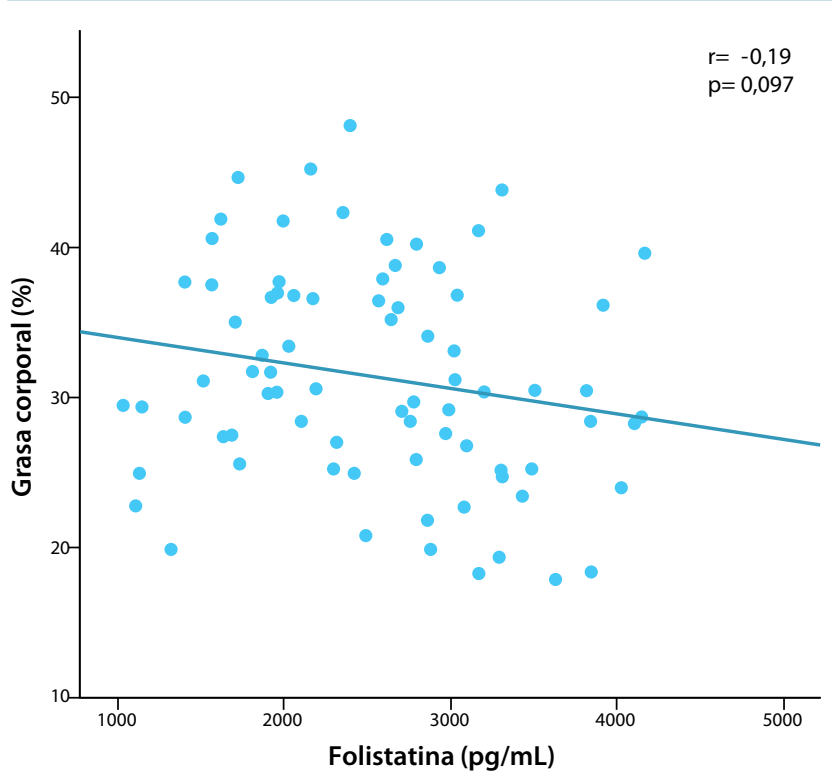

Folistatina y resistencia a la insulina

En la figura 3 se observan los resultados de correlación entre la folistatina y los diversos índices de RI derivados de la PTOG de múltiples puntos. La folistatina no se correlacionó con ninguno de ellos. No encontramos diferencias significativas en características demográficas y clínicas entre el subgrupo sometido al clamp y quienes no participaron del mismo (tabla 2). En el análisis crudo, la folistatina no evidenció correlación con la RI y la captación de glucosa obtenida durante el clamp (M) $(r=0,14, p=0,22)$. Sin embargo, al eliminar un dato poco plausible del análisis, se evidenció una correlación positiva significativa de folistatina con $\mathrm{M}(\mathrm{r}=0,42, \mathrm{p}=0,031)$ (figura 4).

\section{Folistatina y miostatina}

No hallamos asociación entre la concentración de folistatina y miostatina plasmáticas $(\mathrm{r}=0,21, \mathrm{p}=0,064)$. Tampoco entre folistatina plasmática y la relación glucemia/insulinemia basal como subrogado de la relación glucagón/insulina (figura 5).

\section{Discusión}

En nuestro conocimiento, este es el primer estudio publicado acerca de la correlación entre la folistatina plasmática y medidas directas e indirectas de RI en sujetos sin DM. Evaluamos la concentración de folistatina en plasma contra múltiples índices de RI derivados de una PTOG de 5 puntos y contra la captación de glucosa estimulada por insulina en el clamp euglucémico hiperinsulinémico.

Encontramos que la concentración sérica de la folistatina muestra una tendencia hacia una correlación positiva, aunque no significativa, con el porcentaje de masa magra y negativa

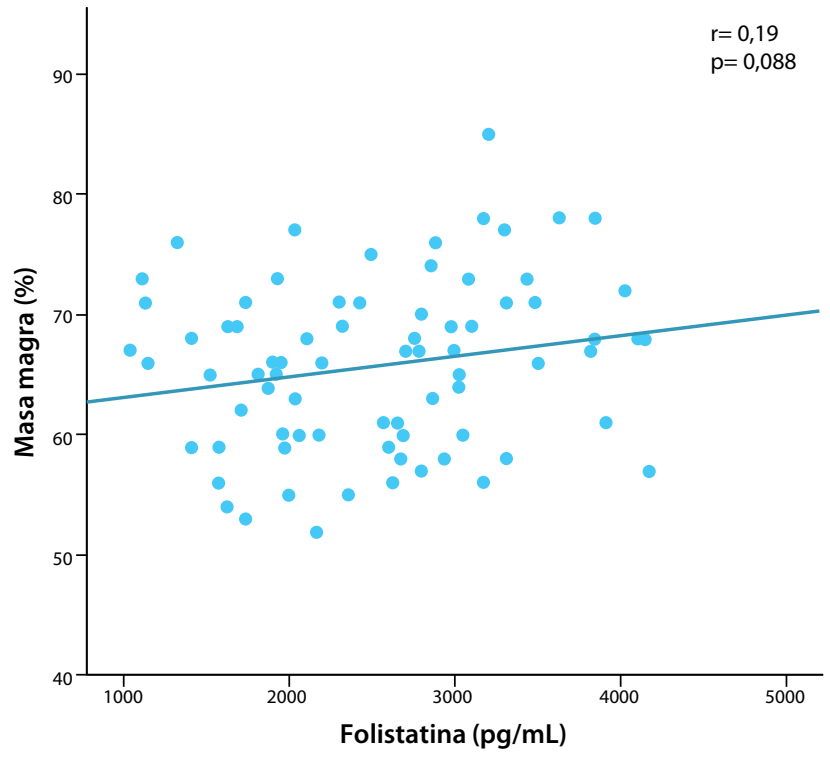

con la adiposidad corporal. A su vez, aunque la folistatina no correlacionó con ninguno de los índices de RI evaluados, sí mostró una correlación positiva con la captación de glucosa derivada del clamp. No encontramos correlación entre las concentraciones séricas de folistatina y miostatina, lo que sugiere el efecto neutralizante de folistatina sobre miostatina no se debe a una reducción en la concentración de esta última, sino que sucede de forma dinámica in vivo. En contraste con reportes previos, no hallamos indicios de asociación entre la relación glucemia/insulinemia basal (como subrogado de la relación glucagón/insulina) y regulación de la secreción de folistatina ${ }^{(1,16,17)}$. Probablemente, la regulación de la producción de folistatina por la relación glucagón/insulina solo llegue a ser relevante en el contexto de la diabetes manifiesta.

De acuerdo con lo descrito por Amthor et $\mathrm{al}^{(8)}$, la folistatina posee la capacidad de antagonizar la acción de la miostatina en la función muscular ${ }^{(22,23)}$. Esto se traduce en la producción de una mayor masa muscular y, por ende, en una menor proporción corporal de tejido adiposo. Lo anterior se relaciona con la tendencia positiva vista en nuestro estudio entre la concentración de folistatina sérica y el porcentaje de masa magra, y la tendencia de correlación negativa con la masa de tejido adiposo corporal.

De forma intuitiva, se espera una correlación negativa entre la concentración de folistatina y miostatina séricas. Sin embargo, según se ha descrito, aunque ambas proteínas se encuentran usualmente en el plasma, no se asocian en dicho medio $^{(8)}$. Esto está acorde con nuestros resultados y posiblemente se debe a que la miostatina circula en forma de complejo, y su concentración en plasma no está directamente relacionada con su acción a nivel muscular ${ }^{(8,24,25)}$. 
Figura 3. Concentración media de folistatina por cuartiles de índices de resistencia a la insulina derivados de la PTOG de 5 puntos
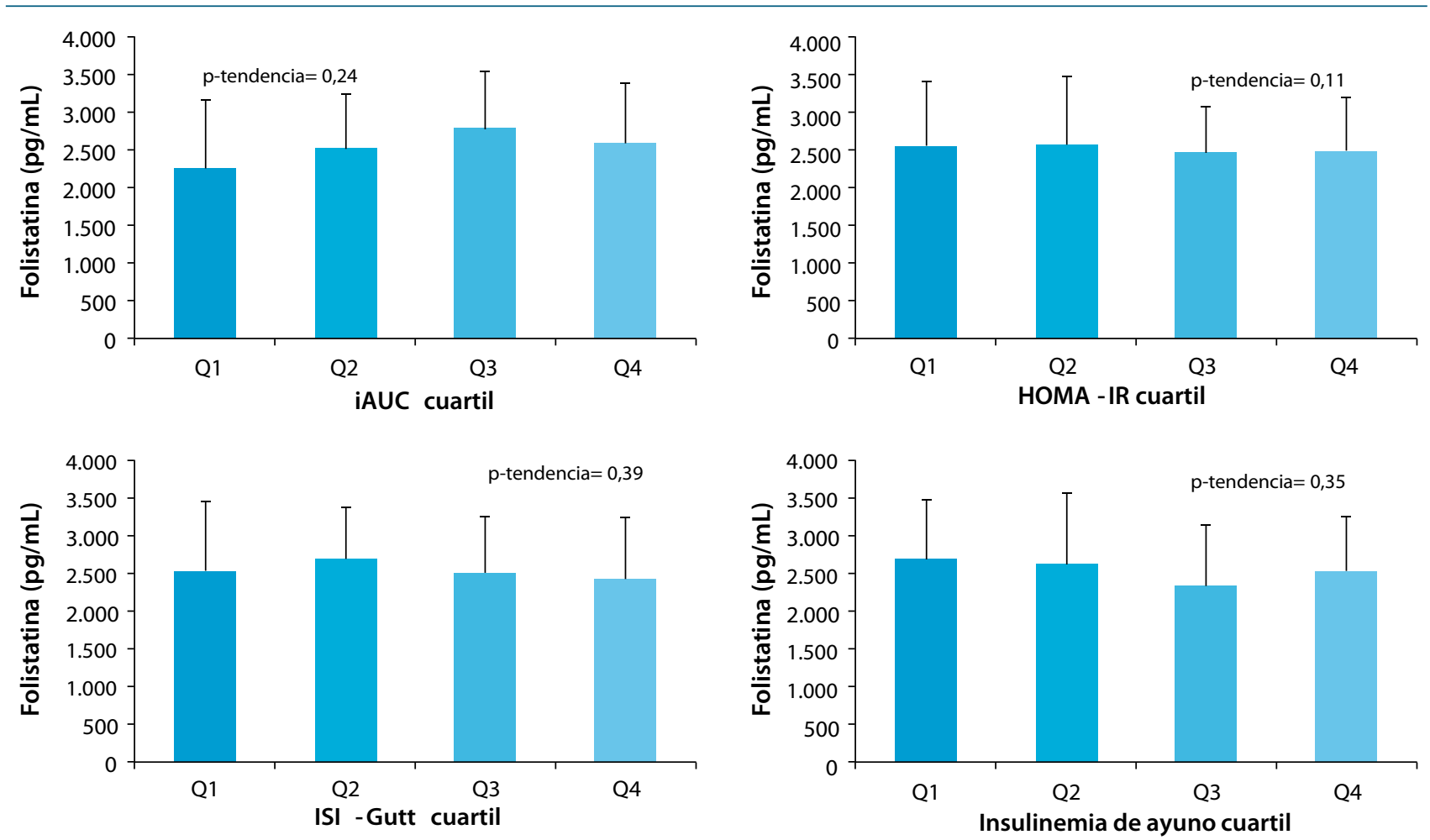

iAUC: área incremental bajo la curva de insulina, HOMA-IR: Valoración de modelo homeostático - insulinorresistencia, ISI-Gutt: Índice de sensibilidad a la insulina según Gutt

Figura 4. Correlación entre folistatina plasmática y captación corporal de glucosa en el clamp hiperinsulinémico euglucémico (M). Resultados con la muestra completa (izquierda) y después de excluir un dato atípico ("outlier") (derecha)

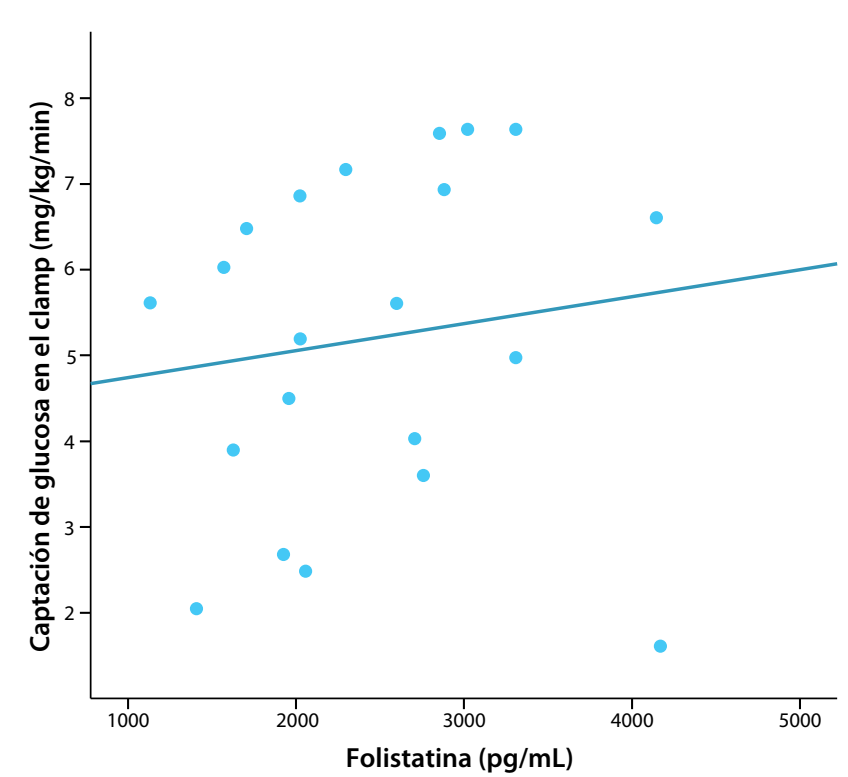

No encontramos estudios publicados que evalúen la asociación entre la folistatina sérica y RI en sujetos sin diagnóstico previo de DM2, pero sí en patologías metabólicas establecidas. La folistatina ha mostrado correlación negativa con el HOMA-

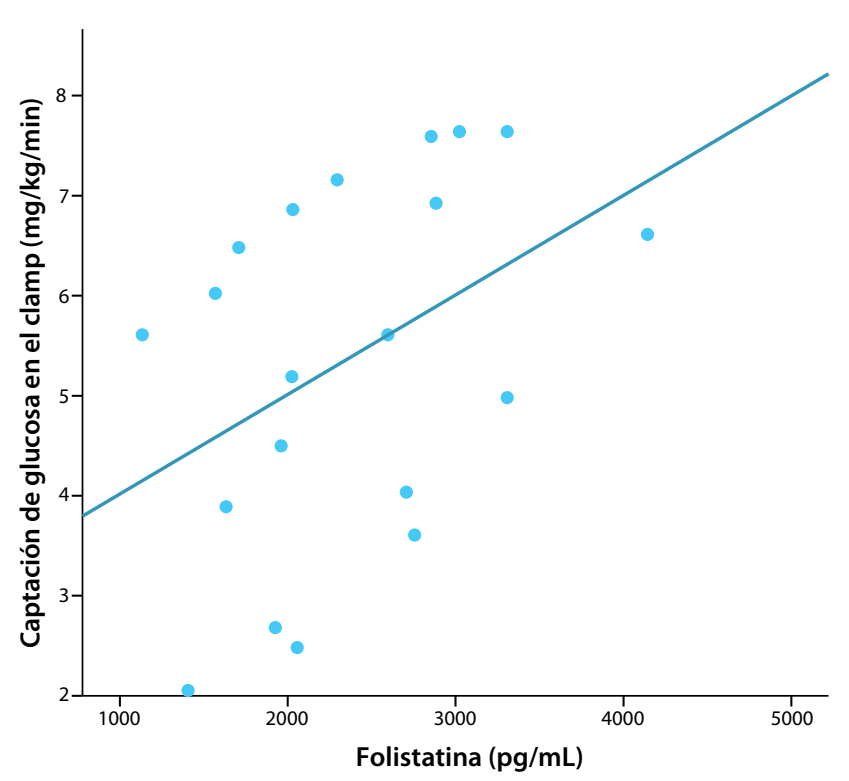

IR en pacientes con diabetes gestacional ${ }^{(26)}$, correlación positiva con el iAUCins y HOMA en síndrome de ovario poliquístico ${ }^{(27)}$, y asociación positiva con HOMA-IR en DM2 ${ }^{(28)}$. En el presente estudio, la folistatina no se correlacionó con los diferentes índices 
Tabla 2. Comparación de las características demográficas, clínicas y de laboratorio de los participantes en el estudio que se sometieron o no al clamp euglucémico-hiperinsulinémico

\begin{tabular}{|c|c|c|c|}
\hline & No Clamp $(n=60)$ & Clamp (n=21) & $\mathbf{p}$ \\
\hline Edad (años) & $52,6 \pm 7,48$ & $51,61 \pm 11,12$ & 0,654 \\
\hline Peso (kg) & $69,97 \pm 10,38$ & $70,06 \pm 15,21$ & 0,977 \\
\hline Estatura (cm) & $162,74 \pm 9,35$ & $161,66 \pm 8,75$ & 0,652 \\
\hline Índice de masa corporal $\left(\mathrm{kg} / \mathrm{m}^{2}\right)$ & $26,32 \pm 2,3$ & $26,69 \pm 4,76$ & 0,648 \\
\hline Agua corporal (\%) & $49,75 \pm 4,39$ & $49,8 \pm 4,98$ & 0,964 \\
\hline Grasa corporal (\%) & $31,37 \pm 7,04$ & $30,98 \pm 7,34$ & 0,836 \\
\hline Grasa abdominal (\%) & $8,62 \pm 2,89$ & $8,95 \pm 4,13$ & 0,694 \\
\hline Masa magra (kg) & $46,31 \pm 9,09$ & $45,78 \pm 9,74$ & 0,823 \\
\hline Masa magra (\%) & $66,1 \pm 6,45$ & $65,82 \pm 7,43$ & 0,908 \\
\hline Masa ósea (kg) & $2,44 \pm 0,46$ & $2,82 \pm 3,08$ & 0,358 \\
\hline Perímetro abdominal (cm) & $86,21 \pm 8,12$ & $88,86 \pm 13,89$ & 0,326 \\
\hline Tensión arterial sistólica (mmHg) & $115,9 \pm 11,48$ & $118,68 \pm 16,52$ & 0,418 \\
\hline Tensión arterial diastólica $(\mathrm{mmHg})$ & $74,65 \pm 9,75$ & $74,47 \pm 11,1$ & 0,948 \\
\hline Glucemia capilar (mg/dL) & $95,48 \pm 6,99$ & $96,08 \pm 10,63$ & 0,772 \\
\hline HbA1c (\%) & $5,37 \pm 0,69$ & $6,62 \pm 6,67$ & 0,180 \\
\hline Creatinina (mg/dL) & $0,83 \pm 0,16$ & $0,81 \pm 0,17$ & 0,621 \\
\hline Colesterol total (mg/dL) & $194,59 \pm 51,34$ & $200,29 \pm 47,49$ & 0,664 \\
\hline Log triglicéridos & $2,17 \pm 1,87$ & $2,2 \pm 1,89$ & 0,568 \\
\hline Colesterol HDL (mg/dL) & $41,13 \pm 10,25$ & $44,98 \pm 12,79$ & 0,180 \\
\hline Colesterol LDL (mg/dL) & $125,43 \pm 46,18$ & $125,02 \pm 44,79$ & 0,972 \\
\hline Proteína C reactiva (g/dL) & $2,64 \pm 1,42$ & $3,27 \pm 4,73$ & 0,462 \\
\hline Glucemia basal (mg/dL) & $95,43 \pm 6,79$ & $96,41 \pm 10,52$ & 0,634 \\
\hline Glucemia - 30 minutos (mg/dL) & $134,57 \pm 21,63$ & $134,16 \pm 37,84$ & 0,953 \\
\hline Glucemia - 60 minutos (mg/dL) & $135,48 \pm 30,44$ & $141,56 \pm 49,08$ & 0,517 \\
\hline Glucemia - 90 minutos (mg/dL) & $116,24 \pm 27,76$ & $129,66 \pm 47,39$ & 0,130 \\
\hline Glucemia - 120 minutos (mg/dL) & $110,52 \pm 19,73$ & $118,66 \pm 46,31$ & 0,280 \\
\hline Insulinemia basal (mUI/dL) & $24,12 \pm 23,9$ & $17,08 \pm 15,43$ & 0,229 \\
\hline Insulinemia - $\mathbf{3 0}$ minutos (mUl/dL) & $67,98 \pm 64,9$ & $72,41 \pm 107,79$ & 0,827 \\
\hline Insulinemia - 60 minutos (mUI/dL) & $81,28 \pm 62,11$ & $72,33 \pm 73,37$ & 0,598 \\
\hline Insulinemia - 90 minutos (mUl/dL) & $60,41 \pm 49,12$ & $49,1 \pm 52,64$ & 0,388 \\
\hline HOMA-IR & $5,62 \pm 5,56$ & $4,1 \pm 3,87$ & 0,270 \\
\hline ISI (Gutt) & $70,61 \pm 17,48$ & $74 \pm 22,49$ & 0,492 \\
\hline QUICKI & $0,32 \pm 0,03$ & $0,33 \pm 0,03$ & 0,240 \\
\hline iAUC-Insulina & $5207 \pm 4840$ & $4724 \pm 6224$ & 0,723 \\
\hline
\end{tabular}

Los datos son expresados como media +/- DS

de resistencia a la insulina derivados de la PTOG de 5 puntos, pero sí evidenciamos una correlación positiva entre la folistatina y la captación de glucosa derivada del clamp hiperinsulinémico-euglucémico, es decir, a mayor sensibilidad a la insulina existe una mayor concentración de folistatina en plasma.

Aunque estos resultados son contradictorios, esta discrepancia entre las mediciones evaluadas de RI de forma directa e indirecta resultan ser un hallazgo interesante. Consideramos que pueden indicar que la folistatina es un marcador de sensibilidad a la insulina, que no se asocia con la RI propiamente dicha. Así, a mayor folistatina sérica, mayor masa muscular y menos tejido adiposo, lo que conlleva a una mejor sensibilidad a la insulina.

Dentro de las limitaciones del presente estudio se encuentran la naturaleza transversal del diseño y un tamaño de muestra relativamente pequeño, que limitan la validez externa de los resultados reportados. No obstante, sí incluimos indivi- 
Figura 5. Concentración plasmática de folistatina de acuerdo con los cuartiles de relación glucemia/ insulinemia de ayuno

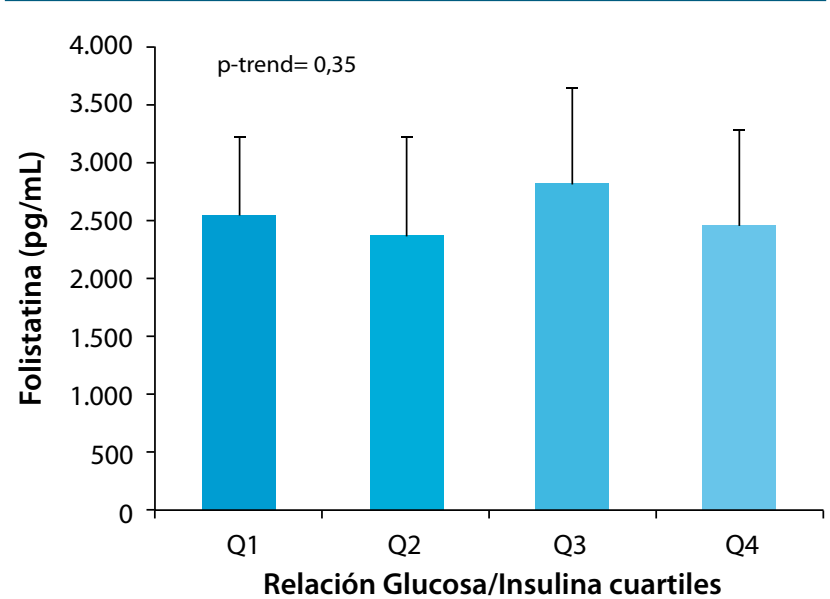

duos de ambos sexos, con una amplia gama de adiposidades, y realizamos diferentes mediciones que reflejan directamente la acción de la insulina y la RI de todo el cuerpo. Además, aunque el grupo sometido al clamp se seleccionó aleatoriamente de la muestra de estudio completa y no fue significativamente diferente de ella, no podemos garantizar que los hallazgos con respecto a la captación de glucosa (M) hubieran sido los mismos en la muestra completa.

\section{Conclusiones}

En este primer estudio en humanos, la folistatina parece correlacionar positivamente con la masa muscular y de forma negativa con el porcentaje de grasa corporal. Además, nuestros hallazgos sugieren que la folistatina es un marcador de sensibilidad a la insulina, pero que no se relaciona directamente con la RI.

\section{Referencias}

1. Hansen JS, Plomgaard P. Circulating follistatin in relation to energy metabolism. Mol Cell Endocrinol. 2016;433:87-93.

2. Hansen J, Rinnov A, Krogh-Madsen R, Fischer CP, Andreasen AS, Berg RM, Møller K, Pedersen BK, Plomgaard P. Plasma follistatin is elevated in patients with type 2 diabetes: relationship to hyperglycemia, hyperinsulinemia, and systemic low-grade inflammation. Diabetes Metab Res Rev. 2013;29:463-72.

3. Ueno N, Ling N, Ying SY, Esch F, Shimasaki S, Guillemin R. Isolation and partial characterization of follistatin: a single-chain Mr 35,000 monomeric protein that inhibits the release of follicle-stimulating hormone. Proc Natl Acad Sci USA. 1987;84:8282-6.

4. Esch FS, Shimasaki S, Mercado M, Cooksey K, Ling N, Ying S, Ueno N, Guillemin R. Structural characterization of follistatin: a novel follicle-stimulating hormone release-inhibiting polypeptide from the gonad. Mol Endocrinol. 1987;1:849-55.

5. Takamura K, Tsuchida K, Miyake H, Tashiro S, Sugino H. Possible endocrine control by follistatin 315 during liver regeneration based on changes in the activin receptor after a partial hepatectomy in rats. Hepatogastroenterology. 2005;52:60-6.

6. Bondestam J, Horelli-Kuitunen N, Hildén K, Ritvos O, Aaltonen J. Assignment of ACVR2 and ACVR2B the human activin receptor type II and IIB genes to chromosome bands 2q22.2-->q23.3 and 3p22 and the human follistatin gene (FST) to chromosome 5q11.2 by FISH. Cytogenet Cell Genet. 1999;87:219-20.

7. Harrington AE, Morris-Triggs SA, Ruotolo BT, Robinson CV, Ohnuma S, Hyvönen M. Structural basis for the inhibition of activin signalling by follistatin. EMBO J. 2006;25:1035-45.

8. Amthor H, Nicholas G, McKinnell I, Kemp CF, Sharma M, Kambadur R, Patel K. Follistatin complexes Myostatin and antagonises Myostatin-mediated inhibition of myogenesis. Dev Biol. 2004;270:19-30.

9. Pentek J, Parker L, Wu A, Arora K. Follistatin preferentially antagonizes activin rather than BMP signaling in Drosophila. Genesis. 2009;47:261-73.

10. Shimasaki S, Koga M, Esch F, Cooksey K, Mercado M, Koba A, Ueno N, Ying SY, Ling N, Guillemin R. Primary structure of the human follistatin precursor and its genomic organization. Proc Natl Acad Sci USA. 1988;85:4218-22.

11. Lerch TF, Shimasaki S, Woodruff TK, Jardetzky TS. Structural and biophysical coupling of heparin and activin binding to follistatin isoform functions. J Biol Chem. 2007;282:15930-9.

12. Schneyer AL, Wang Q Sidis Y, Sluss PM. Differential distribution of follistatin isoforms: application of a new FS315-specific immunoassay. J Clin Endocrinol Metab. 2004;89:5067-75.

13. Vamvini MT, Aronis KN, Chamberland JP, Mantzoros CS. Energy deprivation alters in a leptin- and cortisol-independent manner circulating levels of activin A and follistatin but not myostatin in healthy males. J Clin Endocrinol Metab. 2011;96:3416-23.

14. Hansen J, Brandt C, Nielsen AR, Hojman P, Whitham M, Febbraio MA, Pedersen BK, Plomgaard P. Exercise induces a marked increase in plasma follistatin: evidence that follistatin is a contraction-induced hepatokine. Endocrinology. 2011;152:164-71.
15. Kerschan-Schindl K, Thalmann MM, Weiss E, Tsironi M, Föger-Samwald U, Meinhart J, Skenderi K, Pietschmann P. Changes in Serum Levels of Myokines and Wnt-Antagonists after an Ultramarathon Race. PLoS One. 2015;10:e0132478.

16. Hansen JS, Rutti S, Arous C, Clemmesen JO, Secher NH, Drescher A, GonelleGispert C, Halban PA, Pedersen BK, Weigert C, Bouzakri K, Plomgaard P. Circulating Follistatin Is Liver-Derived and Regulated by the Glucagon-to-Insulin Ratio. J Clin Endocrinol Metab. 2016;101:550-60.

17. Hansen JS, Pedersen BK, Xu G, Lehmann R, Weigert C, Plomgaard P. ExerciseInduced Secretion of FGF21 and Follistatin Are Blocked by Pancreatic Clamp and Impaired in Type 2 Diabetes. J Clin Endocrinol Metab. 2016;101:281625.

18. DeFronzo RA, Tobin JD, Andres R. Glucose clamp technique: a method for quantifying insulin secretion and resistance. Am J Physiol 1979;237:E214-23

19. Mendivil CO, Robles-Osorio L, Horton ES, Hamdy O, Caballero AE. Young Hispanics at risk of type 2 diabetes display endothelial activation, subclinical inflammation and alterations of coagulation and fibrinolysis. Diabetol Metab Syndr 2013;5:37.

20. Gutt M, Davis CL, Spitzer SB, Llabre MM, Kumar M, Czarnecki EM, Schneiderman N, Skyler JS, Marks JB. Validation of the insulin sensitivity index (ISI0,120): comparison with other measures. Diabetes Res Clin Pract 2000;47:177-184

21. Matthews JNS, Altman DG, Campbell MJ, Royston P. Analysis of serial measurements in medical research. Br Med J 1990;27:230-235.

22. Zimmers TA, Davies MV, Koniaris LG, Haynes P, Esquela AF, Tomkinson KN McPherron AC, Wolfman NM, Lee SJ. Induction of cachexia in mice by systemically administered myostatin. Science. 2002;296:1486-8.

23. Amthor H, Christ B, Rashid-Doubell F, Kemp CF, Lang E, Patel K. Follistatin regulates bone morphogenetic protein-7 (BMP-7) activity to stimulate embryonic muscle growth. Dev Biol. 2002;243:115-27.

24. Hill JJ, Davies MV, Pearson AA, Wang JH, Hewick RM, Wolfman NM, Qiu Y. The myostatin propeptide and the follistatin-related gene are inhibitory binding proteins of myostatin in normal serum. J Biol Chem. 2002;277:40735-41.

25. Hill JJ, Qiu Y, Hewick RM, Wolfman NM. Regulation of myostatin in vivo by growth and differentiation factor-associated serum protein-1: a nove protein with protease inhibitor and follistatin domains. Mol Endocrinol. 2003;17:1144-54.

26. Näf S, Escote X, Ballesteros M, Yañez RE, Simón-Muela I, Gil P, Albaiges G, Vendrell J, Megia A. Serum activin A and follistatin levels in gestational diabetes and the association of the Activin A-Follistatin system with anthropometric parameters in offspring. PLoS One. 2014;9:e92175.

27. Teede $\mathrm{H}, \mathrm{Ng} \mathrm{S}$, Hedger M, Moran L. Follistatin and activins in polycystic ovary syndrome: relationship to metabolic and hormonal markers. Metabolism. 2013;62:1394-400.

28. Wu H, Wu M, Chen Y, Allan CA, Phillips DJ, Hedger MP. Correlation between blood activin levels and clinical parameters of type 2 diabetes. Exp Diabetes Res. 2012;2012:410579. 